\title{
An Ultra-low Altitude Lunar Orbiter
}

Submitted to the NASEM Planetary Science Decadal Survey $15^{\text {th }}$ September 2020

\author{
Authors: Elisha Jhoti*, David Paige and Tyler Horvath \\ (UCLA)
}

Co-signatories: Tyler Powell (UCLA)

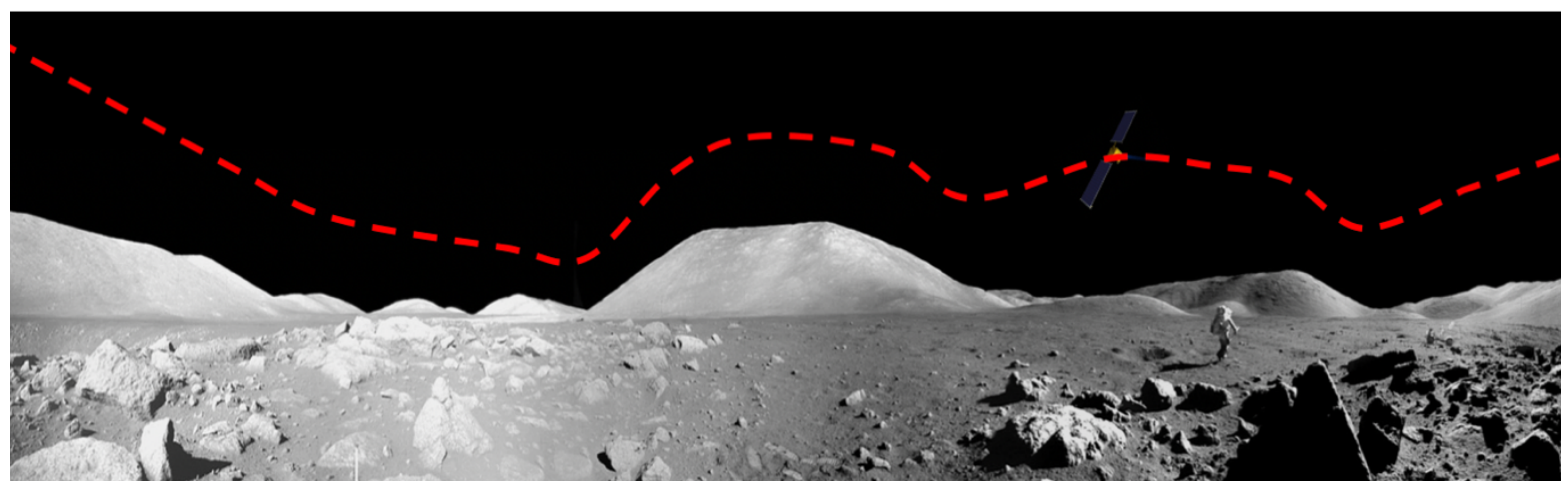




\section{Why do we need an ultra-low altitude lunar orbiter in the next decade?}

The recent LEAG 2019 findings highlighted that orbital datasets with higher resolution than LRO are required for next-decade lunar exploration, which will involve precision targeting of surface assets [1]. There is currently a data resolution gap between orbital datasets and Apollo/Luna surface and sample studies. It is critical to address this in the next decade before the pristine lunar environment is altered with the increasing cadence of planned surface missions [2]. Many lunar orbiters collect their most useful data at the end of their mission, whilst slowly spiraling into the Moon. What if this low altitude flight could be extended for the duration of the mission? An ultra-low altitude lunar orbiter presents enhanced science returns with the possibility of targeted high-resolution datasets at the meter/cm scale.

The Strategic Knowledge Gap Theme 1: Understanding the lunar resource potential from [3] underscored the need for 'data at the $10 \mathrm{~m}$ scale or below over $1-5 \mathrm{~km}$ baselines', i.e. extending orbital data beyond the capabilities of LRO. The demand for higher resolution data is also echoed in the Advancing Science at the Moon - Special Action Team Concepts 3, 4, 7, and 8 [4], as well as in the Scientific Context for Exploration at the Moon [2]. These themes and knowledge gaps could certainly be fulfilled with an ultra-low altitude orbiter, and depending on the instrument payload, probing into the sub-surface may be possible, for example with a ground-penetrating radar on board.

Ultra-low altitude Earth observation has been demonstrated by ESA's Gravity field and steady-state Ocean Circulation Explorer (GOCE), which used a low drag body, accelerometers, and solar electric propulsion to maintain a low Earth orbit [5]. JAXA has also demonstrated an Earth based low altitude spacecraft, the Super Low Altitude Test Satellite (SLATS/ "Tsubame"), which employed agile maneuvers using an ion thruster and gas jet to respond to atmospheric drag [6].

The Moon is an airless body therefore a satellite could achieve extremely low altitudes with hazard avoidance. An agile high-thrust orbiter could approach any point of interest potentially within $10 \mathrm{~km}$ of the surface [7]. This concept is extremely cost-effective as it can be targeted to multiple low altitude locations.

\section{Scientific Motivation}

Many areas of research would benefit from higher resolution data. As an example, we identify three areas where higher resolution data could be utilized: polar ice abundance, resource prospecting for ISRU, and the lunar magnetic environment, including swirls.

LROC's NAC has a spatial resolution of $0.5 \mathrm{~m} / \mathrm{pixel}$ [8]. A low altitude lunar orbiter with a polar orbit could potentially observe down to $\sim \mathrm{cm}$ scale spatial resolution with a highresolution imager payload. This would enable scientific advancements for lunar surface processes and inform future crewed missions to possible ISRU sites, especially in the polar regions. A neutron or gamma ray spectrometer payload could also help fill this gap and inform our understanding of the lunar water cycle and resource distribution. These instruments usually have a large FOV, so they would be less affected by smearing that would impact imagers at this low an altitude. The need for higher resolution orbital data has been emphasized in [1], specifically for resource prospecting for near-future crewed and robotic missions.

Lunar swirls may be explored further at low altitude with a magnetometer. The lowest altitude magnetometer observations of swirls come from the last moments of JAXA's 
Kaguya spacecraft before it crashed into the lunar surface. These data extend below $10 \mathrm{~km}$, as low as $5 \mathrm{~km}$ over Mare Ingenii, revealing observations of the magnetic anomalies that are theorized to cause swirls [9]. Repeated observations at low altitude over multiple swirl regions are required to further probe the structure of these magnetic anomalies.

Table 1 summarizes the various types of scientific investigations that are enabled and/or enhanced by ultra-low altitude orbit capability, depending on payload instruments.

\begin{tabular}{|c|c|c|c|c|c|c|c|c|}
\hline $\begin{array}{l}\text { Scientific } \\
\text { investigation } \\
\text { enabled/enhanced }\end{array}$ & 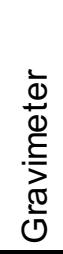 & 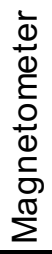 & 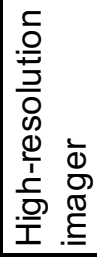 & 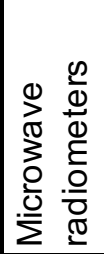 & $\begin{array}{l}\frac{\infty}{\pi} \\
\frac{\pi}{0} \\
\frac{\pi}{\mathscr{r}}\end{array}$ & 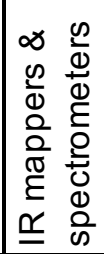 & 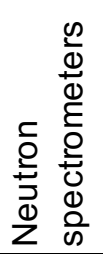 & 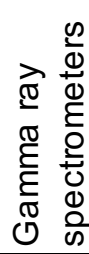 \\
\hline Ice abundance & & & & $x$ & $x$ & $x$ & $x$ & $X$ \\
\hline Surface processes & $x$ & & $\mathrm{x}$ & $x$ & & $x$ & $x$ & $\mathrm{x}$ \\
\hline $\begin{array}{l}\text { Sub-surface } \\
\text { processes }\end{array}$ & $\mathrm{X}$ & & & $\mathrm{x}$ & $x$ & & & \\
\hline ISRU mapping & & & $\mathrm{x}$ & $\mathrm{x}$ & $\mathrm{x}$ & $x$ & $x$ & $\mathrm{X}$ \\
\hline Lunar swirls & & $\mathrm{X}$ & & & & & & \\
\hline $\begin{array}{l}\text { Crewed mission } \\
\text { reconnaissance }\end{array}$ & & & $\mathrm{x}$ & & $\mathrm{x}$ & $x$ & $x$ & $\mathrm{x}$ \\
\hline
\end{tabular}

Table 1: Various types of scientific investigations that could be carried out by potential payload instruments that are enabled and/or enhanced by ultra-low altitude flight.

\section{Enabling technology}

The dominant forces acting on a spacecraft at low altitude on the Moon are from variations in the lunar gravitational field (lunar mascons). These must be mitigated by an ultra-low altitude lunar orbiter, unlike at the Earth where a low altitude orbiter contends primarily with atmospheric drag. An ultra-low altitude orbiter at the Moon would counteract orbital perturbations due to lunar mascons and navigate topography. The spacecraft would operate autonomously with precision accelerometers and thrusters, utilizing gravity and topography data to maintain a near-circular polar low altitude orbit.

\section{Orbital algorithm}

For this paper, an algorithm was written to investigate the feasibility of this method. The orbital mechanics algorithm uses the Verlet Velocity Scheme described in [10] to simulate the spacecraft motion. The algorithm is widely used due to its high precision and minimal computing requirements [10].

The gravitational field of a planetary body can be described using an expansion of spherical harmonics to degree $(l)$ and order $(m)$. NASA's Gravity Recovery and Interior Laboratory (GRAIL) mission measured the Moon's gravitational field to a very high spatial resolution $(l \geq 1200)$. This ensured the smallest scale structures within the gravitational field were accurately captured. To incorporate gravity into the integration scheme, the software package SHTOOLS, developed by [11], along with GRAIL data [12], was used to determine the acceleration acting on the spacecraft at each position. SHTOOLS produces global maps of 
gravity accelerations for any altitude from the spherical harmonic coefficients of the gravitational field. SHTOOLS calculates gravitational potential, $V$, as

$$
V=\frac{G M}{r} \sum_{l=0}^{l_{\max }}\left(\frac{r_{0}}{r}\right)^{l} \sum_{m=-l}^{l} C_{l m} Y_{l m}
$$

Where $M$ is the Moon's mass, $r 0$ is the Moon's radius, $r$ is the spacecraft's distance from the Moon's center $\left(r O+\right.$ spacecraft altitude), $l$ is the degree and $m$ is the order of the field, $C_{l m}$ are the spherical harmonic coefficients provided by the GRAIL data, and $Y_{l m}$ are the associated normalized Legendre polynomials. SHTOOLS then converts gravitational potential to gravitational acceleration, $B$, using $B=\nabla V$ [11]. For this algorithm, the global map of gravity accelerations produced by SHTOOLS is initialized each time the satellite's altitude strays outside of a $\pm 10 \mathrm{~m}$ station-keeping altitude deadband to reduce computing requirements.

Data from NASA's Lunar Orbiter Laser Altimeter (LOLA) instrument [13] were used to determine the spacecraft's height above the lunar surface and whether its trajectory would intersect the surface. The topography data were in the equidistant cylindrical projection with a resolution of $16 \mathrm{ppd}$, spanning the latitude range $\left(-90^{\circ}, 90^{\circ}\right)$ and longitude range $\left(0^{\circ}, 360^{\circ}\right)$.

\section{Algorithm results}

The algorithm was run with a spherical harmonic expansion for gravity to $l=660$ degrees in order to capture the smaller-scale variations in the gravitational field. The spacecraft was given a starting altitude of $10 \mathrm{~km}$, a starting position of latitude $=88^{\circ}$, longitude $=0^{\circ}$ and a launch angle of $15^{\circ}$. Figure 1a shows the altitude of the spacecraft over $\sim 1.5$ orbits with the topography from LOLA [13] at each spacecraft position plotted below it. Interestingly the spacecraft did not intersect the surface but likely would if the integration had been run for longer. This indicates that active propulsion is required to maintain a stable orbit at low altitude.

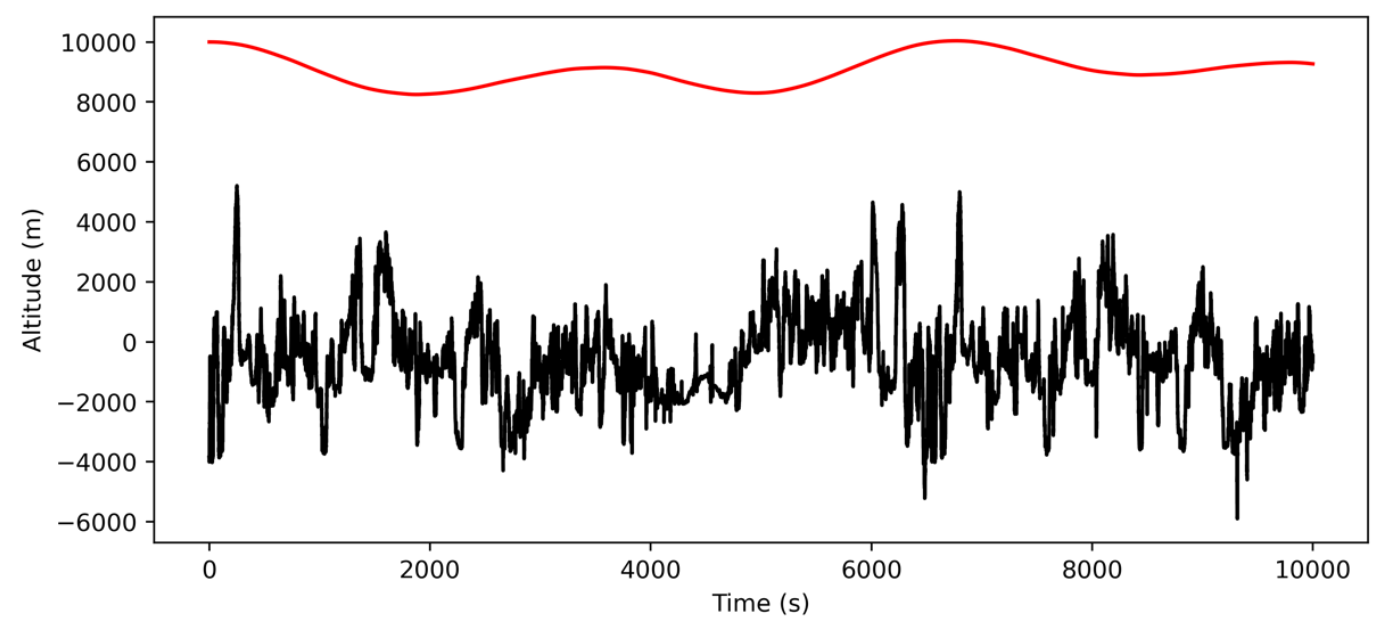

Figure 1a: Plot showing interpolated height values from LOLA data (black) with the spacecraft altitude relative to the reference radius of $1738 \mathrm{~km}$ along $\sim 1.5$ orbits (red). The spacecraft orbit was started at latitude $=88^{\circ}$, longitude $=0^{\circ}$, altitude $=$ $10 \mathrm{~km}$, launch angle $=15^{\circ}$. 


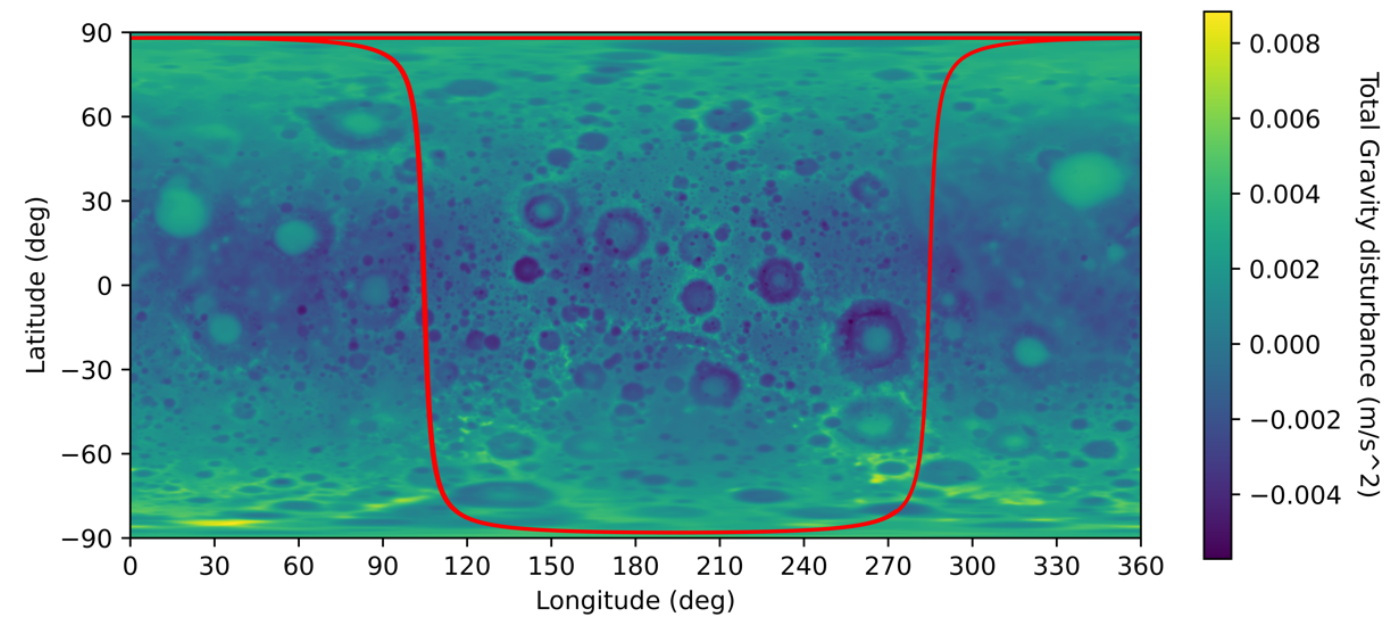

Figure 1b: Global map of total gravity disturbance at $10 \mathrm{~km}$ altitude above the reference radius of $1738 \mathrm{~km}$. Red line shows the spacecraft positions along $\sim 1.5$ orbits starting at latitude $=88^{\circ}$, longitude $=0^{\circ}$, altitude $=10 \mathrm{~km}$, launch angle $=15^{\circ}$, (the same orbit as in Figure 1a).

\section{Propulsion \& Thrusters}

The ultra-low altitude orbiter will use gravity and topography data knowledge to predict the gravitational variations that will be acting on the spacecraft based on its exact position, using an algorithm similar to the one we have developed. The spacecraft will then employ agile thrusters to mitigate these forces and avoid topographic changes.

Due to the significant gradients in topography and need for constant station-keeping, mass efficiency and high specific impulse of a potential propulsion system will need to be prioritized. Further investigation is required to determine a sufficient propulsion system, such as a comparison between chemical propulsion and solar electric propulsion.

\section{Cross-cutting themes}

Ultra-low altitude orbiters are an enabling technology for any instrument that could benefit from low altitude measurements. The Moon is an excellent proving ground for ultra-low altitude technology as there is no atmosphere. An ultra-low altitude orbiter at Mars has been explored and proposed by [14], but this will have to contend with atmospheric drag and utilize a high precision atmospheric model. Therefore, demonstrating ultra-low altitude flight at the Moon would pave the way for an orbiter at Mars and other planetary bodies.

\section{References}

[1] https://www.lpi.usra.edu/leag/meetings/2019 Findings Final.pdf

[2] The Scientific Context for Exploration at the Moon, (2007), Ch.3, Table 3.1, Science Concept 8.

[3] LEAG Lunar Human Exploration Strategic Knowledge Gap Special Action Team Review (Sept 2016).

[4] https://www.lpi.usra.edu/leag/reports/ASM-SAT-Report-final.pdf

[5] Drinkwater, M. R. et al. (2003) Space Sciences Series of ISSI Vol. 17.

[6] "Tsubame Transition to Orbit Keeping Operations", JAXA Press Release, $18^{\text {th }}$ March 2019.

[7] Jhoti, E. et al. LPSC 2020 Abstract \#2480.

[8] Robinson, M. S. et al. (2010) Space Sci Review Vol 150 p.81-124.

[9] Saito, Y. et al. (2019) AGU Proceedings \#P33C-02.

[10] Anzalone, E. and Chai, P. (2015), AE 6354 Advanced Orbital Mechanics, doi: 10.13140/RG.2.1.1474.2243

[11] Wieczorek, M. A. et al (2018), Geochemistry, Geophysics, Geosystems, 19, 2574-2592.

[12] Lemoine, F. G. et al (2013) JGR Planets, 118, 1676-1698.

[13] Neumann, G.A., 2009 Lunar Orbiter Laser Altimeter Raw Data Set, LRO-L-LOLA-4-GDR-V1.0, NASA PDS, 2010.

[14] Paige, D. A. et al (2019) AGU Fall Meeting 2019, Abstract \#P33D-16.

Cover page photo credit: Apollo 17 landing site https://www.hq.nasa.gov/alsj/a17/a17.1464906_gpan.jpg, NASA DART spacecraft NASA/JHUAPL. 